\title{
Rare case of mitral annulus disjunction and noncompaction-like myocardium
}

\author{
Bryan E-Xin Tan ${ }^{1}$, Mallory Balmer-Swain², Mohan Rao ${ }^{2}$, Bipul Baibhav² \\ ${ }^{1}$ Department of Internal Medicine, Rochester General Hospital, Rochester, NY, USA \\ ${ }^{2}$ Sands Constellation Heart Institute, Rochester Regional Health, Rochester, NY, USA
}

\begin{abstract}
A 74-year-old man presented to the cardiology office with exertional dyspnea. Transthoracic echocardiography showed mild left ventricular (LV) dilation, LV hypertrophy, LV ejection fraction $50 \%$, mitral valve prolapse and bicuspid aortic valve. Stress echocardiography was nonrevealing. Coronary angiography showed non-obstructive coronary artery disease. Cardiac magnetic resonance imaging was performed, and showed a $9.1 \mathrm{~mm}$ atrial displacement of the posterior mitral valve leaflet at the hinge point during systole; the finding was consistent with mitral annulus disjunction (Fig. 1A, Suppl. Video 1). Cardiac magnetic resonance imaging also showed spongy/noncompacted myocardium in the LV, with a noncompaction-to-compaction ratio of 1.86 during end-diastole (Fig. 1B). Late gadolinium enhancement images did not show myocardial fibrosis or presence of LV thrombus. 24-hour Holter monitoring showed occasional premature ventricular complex, but no ventricular tachycardia.
\end{abstract}

Mitral annulus disjunction and LV noncompaction are rare arrhythmogenic cardiac anomalies. Mitral annulus disjunction is most commonly seen in patients with mitral valve prolapse. Mitral annulus disjunction distance $>8.5 \mathrm{~mm}$, and myocardial fibrosis of LV papillary muscles and basal inferior wall are high risk features for ventricular arrhythmias. For patients with $\mathrm{LV}$ noncompaction, $\mathrm{LV}$ systolic impairment is associated with increased risk of sudden cardiac death. Competitive sports should be refrained in individuals with LV noncompaction and systolic impairment.

According to the current guidelines, implantable-cardioverter defibrillator is not indicated in patients with mitral annulus disjunction or LV noncompaction unless there is a history of sustained ventricular tachycardia, ventricular fibrillation, or aborted sudden cardiac death. To our best knowledge, this is the first reported case describing a patient with concomitant mitral annulus disjunction and LV 'spongy' noncompaction-like myocardium on cardiac magnetic resonance imaging.

Conflict of interest: None declared

Address for correspondence: Bryan E-Xin Tan, MD, 1425 Portland Avenue, Rochester, NY, 14621, USA, tel: +1(585)922-4365, e-mail: Bryan.Tan@rochesterregional.org

Received: 25.04.2020 Accepted: 5.05.2020 


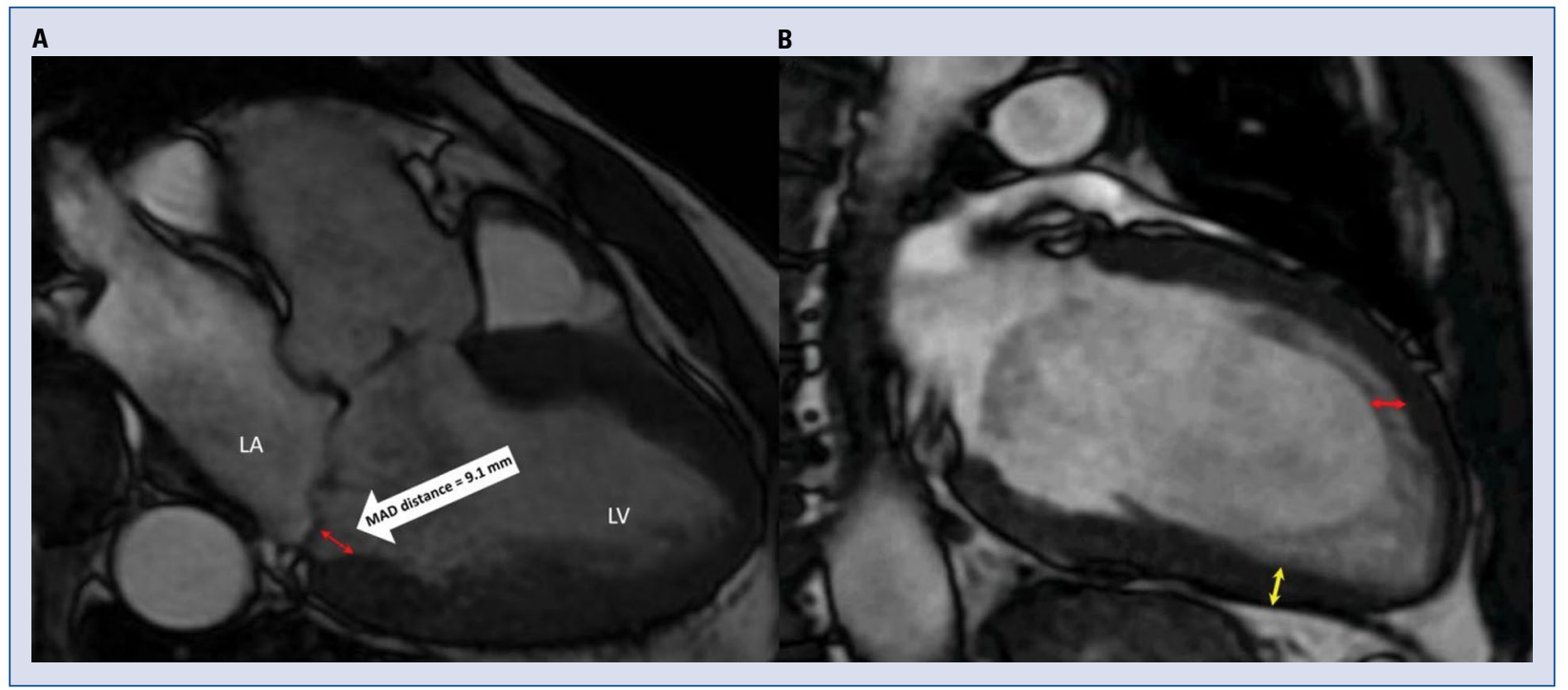

Figure 1. A. Cardiac magnetic resonance imaging (MRI) showed mitral annulus disjunction (MAD) with MAD distance of $9.1 \mathrm{~mm}$ during systole (red arrow); B. Cardiac MRI showed noncompacted myocardium at the apical cap, all apical segments, and mid-lateral wall with noncompacted to compacted myocardial thickness ratio of 1.86 during end-diastole. Red arrow — noncompacted myocardium; yellow arrow — compacted myocardium; LA — left atrium; LV - left ventricle. 